\title{
Percepción actual y perspectiva del sistema nicaragüense de investigación e innovación agropecuaria
} $2019-2021$

\section{Current Perception and perspective of the Nicaraguan system of agricultural research and innovation $2019-2021$}

\author{
Pedro Pablo Benavídez Torres ${ }^{1,}$ Jairo Rojas Meza \\ ${ }^{1}$ Delegado Regional, ORCID: https://0000-0001-7622-5597 / pebena@yahoo.com, Instituto Nicaragüense de Tecnología Agropecuaria / \\ CNIA Managua. \\ ${ }^{2}$ Docente - Investigador, ORCID: https://0000-0003-2231-4054 / jrojas_12@yahoo.com, Universidad Nacional Autónoma de Nicaragua \\ UNAN Managua / FAREM Matagalpa
}

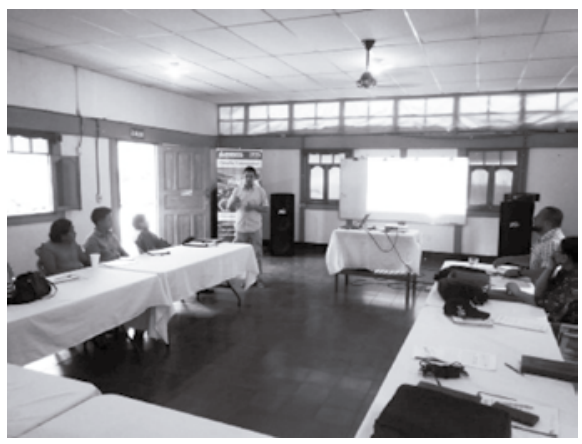

\section{RESUMEN}

El presente artículo es el resultado de una investigación que se realizó en el año 2019 con 24 actores del sector público, sector científico - tecnológico y sector productivo que han participado del Sistema Nicaragüense de Investigación e Innovación Agropecuaria SNIA a partir de su instalación en el año 2015 sobre las percepción actual con relación a lo actuado y estado del conocimiento durante el período 2015 - 2018 y las principales valoraciones, expectativas, perspectivas y prioridades del mismo, para el período 2019 - 2021 . La investigación permitió conocer las principales auto valoraciones del SNIA en términos de su gestión, implementación y desempeño con un resultado que no supera el percentil 70 como mínimo esperado para un SNIA aceptable, así mismo se comparten las principales brechas positivas a partir del estado actual vs estado esperado a partir de una medición de 57 indicadores para el estado actual y los mismos 57 indicadores para la percepción esperada que permitieron derivar las prioridades y perspectivas del SNIA del 2019 al 2021. El tipo de investigación es descriptiva y retro-prospectiva, la escala utilizada fue construida a partir de los criterios e indicadores para medir innovación desde los territorios. Se utilizaron como estrategia de recolección de datos las técnicas de la encuesta, entrevista, revisión documental y la observación con actores claves del SNIA.

Palabras clave: prioridades, brechas positivas, perspectivas Abreviatura: SNIA: Sistema Nicaragüense de Investigación e Innovación Agropecuaria.

\section{ABSTRACT}

This article is the result of a research conducted in 2019 with 24 actors from the public sector, scientific-technological sector and productive sector that have participated in the Nicaraguan System of Agricultural Research and Innovation SNIA from its installation in 2015 on the current perception in relation to the actions and State of knowledge during the period 2015 - 2018 and the main assessments, expectations, perspectives and priorities of the same, for the period $2019-2021$. The research allowed us to know the main self-evaluations of the SNIA in terms of its management, implementation and performance with a result that does not exceed the 70th percentile as minimum expected for an acceptable SNIA, as well as sharing the main positive gaps from the current state vs. expected state from a measurement of 57 indicators for the current state and the same 57 indicators for the expected perception that allowed us to derive the priorities and perspectives of the SNIA from 2019 to 2021. The type of research is descriptive and retro-prospective, the scale used was constructed from the criteria and indicators to measure innovation from the territories. As a data collection strategy, the techniques of survey, interview, literature review and observation with key actors of the SNIA were used.

Keywords: priorities, positive gaps, perspectives

Abbreviations: SNIA (by its acronym in Spanish): Nicaraguan System of Agricultural Research and Innovation.
Recibido: 2 de julio del 2019

Aceptado: 30 de septiembre del 2019

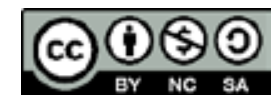




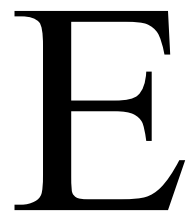

1 Sistema Nacional de Innovación de Nicaragua (SNIN) comprende un complejo grupo de actores del sector productivo (empresas de bienes y servicios), organizaciones públicas dependientes del Gobierno Central y de los gobiernos departamentales, universidades y centros de investigación, organizaciones no gubernamentales (ONG) la sociedad civil. Estos actores interactúan para compartir información y eventualmente conocimiento, además de intercambiar recursos físicos, con el fin de contribuir de diferentes formas a la creación y uso de conocimiento para el desarrollo de la agricultura en Nicaragua. Estas interacciones impactan también en la formación y evolución de las instituciones económicas y políticas del país, que influyen a su vez en la toma de decisiones con respecto al uso y generación de ciencia, tecnología e innovación (CONICYT, 2010, p. 29).

$\mathrm{Al}$ respecto, el Sistema Nicaragüense de Investigación e Innovación Agropecuaria (SNIA) se instaló el 13 de febrero 2015 con la participación de 249 participantes representados por el Instituto Nicaragüense de Tecnología Agropecuaria (INTA), universidades miembros del Consejo Nacional de Universidades (CNU), Instituciones del Sistema Nacional Producción Consumo y Comercio (SNPCC), Organizaciones de productores/as tales como: Consejo Nacional de Cooperativas (CONACOOP), Federación de Asociaciones Ganaderas de Nicaragua (FAGANIC), Asociación Nicaragüense de Productores de Sorgo (ANPROSOR), Unión Nacional Agropecuaria de Productores y Asociados (UNAPA), Asociación Nicaragüense de Arroceros (ANAR), Comisión Nacional Ganadera de Nicaragua (CONAGAN), Movimiento de Productoras y Productores Agroecológicos y Orgánicos de Nicaragua (MAONIC); centros de investigación nacional e internacional y miembros de la cooperación. Dentro de los resultados logrados por el SNIA es mínimo lo que se ha logrado medir más allá de la organización y su funcionamiento durante el 2015 - 2019.

Por lo anterior, se ha planteado en esta investigación la necesidad de comprender cuál es el estado actual de la percepción y perspectivas de los diferentes actores que conformaron el SNIA en el año 2015, tomando como factores de autoevaluación la Innovación a nivel de Gestión, Implementación y Desempeño del SNIA; los Impactos potenciales del SNIA en las dimensiones social, económica, ambiental y organizacional; y las principales prioridades del SNIA a partir del año 2019 al 2021.

Finalmente, con los resultados de la presente investigación se procura contribuir a la continuidad y fortalecimiento del SNIA, mediante la generación de cono- cimientos y propuestas que permitan al INTA retomar el SNIA e impulsarlo con prioridades del corto plazo y visión de mediano y largo plazo.

\section{MATERIALES Y MÉTODOS}

De acuerdo al método de investigación el presente estudio es observacional y según el nivel inicial de profundidad del conocimiento es descriptivo (Piura López, 2012). Según la clasificación de (Hernández, Fernández y Baptista, 2014) , el tipo de estudio es correlacional. En cuanto al tiempo de ocurrencia de los hechos y registro de la información, el estudio es retro-prospectivo, por el período y secuencia del estudio es transversal y según el análisis y alcance de los resultados el estudio es analítico (Canales, Alvarado, y Pineda, 1994). La escala utilizada fue construida a partir de los criterios e indicadores para medir innovación desde los territorios (Rojas Meza, 2015, p. 47).

El tipo de muestreo es no probabilístico y el principal criterio para la selección de la muestra ha sido que los encuestados debían ser actores miembros del SNIA en cualquier estamento u espacio de actuación o participación tanto al nivel territorial, regional como nacional. Otro criterio muy importante ha sido la disposición de los actores a ser entrevistados, y a brindar información de la manera más diáfana posible.

Para la consecución de los objetivos de la investigación se utilizaron diferentes técnicas de recolección de datos, tales como la encuesta, entrevista, observación y revisión documental con actores claves del SNIA.

La entrevista se aplicó al equipo de coordinación nacional del SNIA conformado por el INTA, Consejo Nacional de Universidades CNU y Consejo Nacional de Cooperativas CONACOOP; así mismo se hicieron tres entrevistas más a otros actores claves en la implementación del SNIA como son FAO, CATIE y CRS.

La encuesta se dirigió a 90 personas de 28 actores del SNIA pero solo respondieron 63 de los 90; de los cuales el $75 \%$ son hombres, y en cuanto a la edad mínima de los encuestados es de 29 años y la máxima de 69 años con una mediana de 51 años, y la mayor parte de los encuestados se concentraron entre las edades de 41 años para el percentil 25 y 57 años para el percentil 75, distribuidos en 28 personas del Sector Público, 26 personas del Sector Científico Tecnológico y nueve personas del Sector Productivo.

La observación se desarrolló durante el proceso del estudio, en cuanto a la iniciativa y reacciones ante la solicitud de participar del proceso del estudio, considerándolo con un enfoque de autoevaluación dado que los consultados son miembros del SNIA y muchos de ellos no respondieron la encuesta o bien decidieron no participar del estudio. 
Para el análisis estadístico se desarrollaron tres pasos fundamentales, inicialmente se ha hecho el análisis del Alfa de Cronbach y comparación con escala de confiabilidad del 97.3\%; en un segundo momento se han elaborado 18 perfiles, 9 brechas positivas y 2 perfiles de percepción global del SNIA partiendo de los 9 factores o variables en estudio, y finalmente se han logrado hacer inferencia o contraste de hipótesis.

El formato de la escala Likert utilizado contiene un formato con doble respuesta; una para la percepción actual del indicador afirmado; y otra respuesta para el estado deseado o perspectiva personal o institucional sobre dicho indicador de acuerdo al valor que cada encuestado le dio según su relevancia o pertinencia para él en su contexto y alcances como actor del SNIA. La escala está contenida por cinco categorías de 1 a 5 que corresponden 1-Totalmente En Desacuerdo (TED), 2-En Desacuerdo (ED), 3-Neutral (N), 4-De Acuerdo (DA), 5-Totalmente De Acuerdo (TDA).

Se desarrolló un análisis estadístico para la inferencia o contraste de hipótesis, mediante la comparación de medias previamente agrupadas por factor para correr las pruebas $\mathrm{T}$ para una muestra, obteniendo medias que al compararlas con el valor descrito rápidamente según el intervalo de confianza se puede concluir en términos de Rechazo o No Rechazo de Ho.

Finalmente, se presentan las prioridades del SNIA 2019 - 2021 partiendo del análisis de los diferentes factores, triangulado con la percepción de las entrevistas obtenidas de actores claves en el SNIA y la experiencia del investigador en el tema.

\section{RESULTADOS Y DISCUSIÓN}

Innovación a nivel de Gestión, Implementación y Desempeño del Sistema Nicaragüense de Investigación e Innovación Agropecuaria SNIA. El proceso de globalización produce importantes cambios en la geografía de la producción, no sólo manufacturera, sino en su acepción más amplia, incluyendo actividades como la agricultura y el turismo. La libre circulación del capital en nuevos espacios ampliados de comercio y los procesos de reconversión a los que se ven empujados los territorios, sumados a las innovaciones tecnológicas, generan nuevos mapas productivos, con sus inevitables balances de pérdidas y ganancias (Boisier, 2005, p. 61).

En la gestión del SNIA se espera en los territorios una mayor dinámica del capital social, humano y económico que faciliten los procesos de innovación. A nivel de la implementación dichos procesos de innovación territo-
}

riales deberían ser diseñados e implementados considerando las demandas y/o necesidades de los distintos actores del sector agropecuario y finalmente el desempeño del SNIA debería apuntar a que estos procesos de innovación implementados en los territorios contribuyan a elevar la productividad y competitividad del sector agropecuario con impactos económicos, sociales y ambientales.

Comportamiento de los indicadores sobre la percepción actual 2019 del Sistema Nicaragüense de Investigación e Innovación Agropecuaria SNIA. En la percepción actual 2019 del SNIA, el nivel máximo representa un SNIA ideal; el nivel teórico es lo que al menos se debe lograr para ser un SNIA aceptable; lo real es la percepción 2019 de los actores y el límite inferior es lo peor que puede llegar a ser el SNIA, siendo el factor sobre la Relación Beneficio Costo producto de la interacción en el SNIA en donde la percepción se acerca a dicho límite inferior, por cuanto es donde más se debe enfocar las estrategias o líneas de trabajo del SNIA en términos de impactos y su medición.

Para el Factor Total Percepción Actual 2019 (retrospectiva 2015-2019) se realizó formulación de hipótesis tomando como base un percentil 70 (mínimo esperado), al realizar las pruebas $\mathrm{T}$ para una muestra se encontró diferencias altamente significativas en la percepción total de la Gestión, Implementación y Desempeño del SNIA con relación al período $2015-2019$ (t: -6.268; p-valor=0.000).

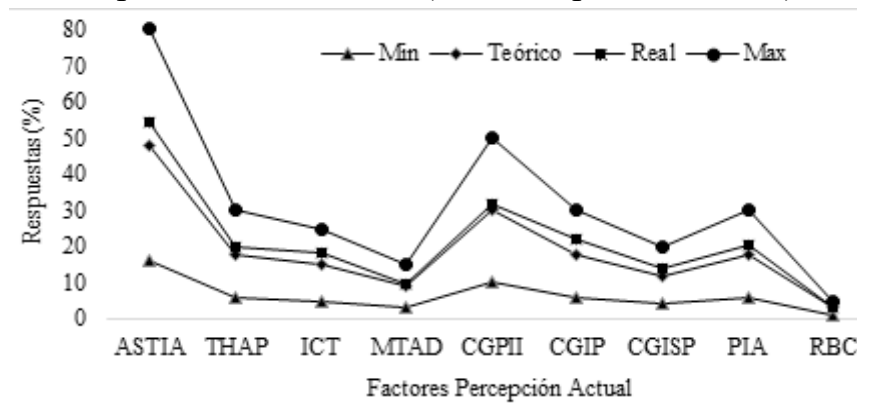

Figura 1. Percepción actual del Sistema Nicaragüense de Investigación e Innovación Agropecuaria SNIA 2019.

ASTIA = Articulación del Sistema Territorial de Innovación Agropecuario, THAP=Talento Humano en las Áreas Agropecuarias Prioritarias, $\mathbf{I C T}=$ Infraestructura Científica Tecnológica, $\mathbf{M T A D}=$ Mercados de Tecnologías Apropiadas y Disponibles, CGPII= Calidad en la gestión de los procesos de innovación de las instituciones de formación e investigación agropecuaria y áreas afines, $\mathbf{C G I P}=$ Calidad en la gestión de la innovación desde el sector público, CGISP= Calidad en la gestión de la innovación en el sector de productores, PIA= procesos de innovación agropecuaria, $\mathrm{RBC}=$ Relación beneficio costo de los procesos de innovación agropecuaria. 
La gestión exitosa del SNIA está determinada por dos factores claves, siendo el de mayor importancia el referido a la articulación del Sistema Territorial de Innovación Agropecuario, seguido de la calidad en la gestión de los procesos de innovación de las instituciones de formación e investigación agropecuaria y áreas afines; en contraste el factor que no está representando al SNIA en su desempeño es la relación beneficio costo de los procesos de innovación con una percepción mínima, así mismo en la implementación el factor que no refleja éxito del SNIA es la calidad en la gestión de la innovación en el sector de los productores destacándose una percepción muy por debajo del nivel teórico que sería lo más aceptable pero no lo exitoso esperado para el SNIA.

A pesar de que los sistemas de innovación territorial son altamente relevantes, el SNIA aún no muestra los aportes exitosos esperados o resultantes producto del triángulo científico tecnológico en el sector agropecuario desde el punto de vista económico y ambiental para los diferentes actores, siendo coherente con la baja gestión de la innovación del sector productivo; lo que significa que el SNIA aún mantiene una gestión más a nivel organizativo sin evolucionar a indicadores de más impactos tangibles en el sector productivo del país.

Lo anterior destaca un punto de partida para el SNIA en donde la innovación continúa siendo un reto importante para el sector agropecuario de Nicaragua.

La innovación en productos, en procesos y en gestión impacta positivamente el crecimiento del empleo y las ventas, y así mismo a las utilidades. Estos resultados son especialmente relevantes para los países de la Alianza del Pacífico (AP) y pueden ayudar a los empresarios, a los gobiernos y a la academia para impulsar acciones que favorezcan la innovación de las empresas y su competitividad (García, Gálvez, y Maldonado, 2016, p. 326).

Desde el punto de vista de la interacción de los mercados de tecnologías apropiadas y disponibles, el SNIA aún no percibe avances importantes en este factor; continúa siendo un desafío la superación de brechas tecnológicas que afectan al sector productivo según sus necesidades tecnológicas productivas en los diferentes territorios.

La ausencia en nuestro país de un sistema articulador entre los investigadores, los extensionistas y los productores es una de las más graves deficiencias que padece nuestro sector, frente a los desafíos y retos de mejorar la productividad, calidad y rentabilidad de los sistemas productivos a través de la innovación (Briones, et al. 2015, p. 5).
Por esta razón se plantea el establecimiento de relaciones solidarias de mercado para generar un efecto multiplicador que facilite las nuevas tecnologías y servicios a un mayor número de pequeños y medianos productores del país, garantizando la sostenibilidad de las innovaciones mismas.

En este sentido, el INTA avanza en una estrategia de bioinsumos para dinamizar la oferta de tecnologías que reduzcan esta brechas entre las necesidades del sector productivo y el acceso a tecnologías apropiadas y disponibles; no obstante la percepción actual del SNIA referente a este indicador de mercados tecnológicos confirma la demanda, pero también indica que hay que redefinir la estrategia como Sistema de Innovación del país en función de más de 20 rubros productivos y diferentes ejes temáticos como la variabilidad climática.

Las alianzas para el desarrollo tienen el potencial de facilitar este marco de actuación creando sinergias entre las contribuciones de cada aliado, optimizando su asignación de recursos y facilitando el logro de resultados concretos (Casado-Cañeque, 2007, p. 6).

\section{Prospectiva 2019 - 2021 del Sistema Nicaragüense de Investigación e Innovación Agropecuaria SNIA. En la} teoría Prospectiva el futuro no lo construye el hombre individual, sino el hombre colectivo que son los "Actores Sociales". Los Actores Sociales son grupos humanos que se unen para defender sus intereses y que obran utilizando el grado de poder que cada uno puede ejercer. Las tendencias (sociales, económicas, etc.) existen porque han sido el fruto de estrategias desplegadas por Actores Sociales. Y si las rupturas a estas tendencias no han logrado hacerlas cambiar su rumbo, ha sido porque el poder de estos actores sociales no sido suficientemente fuerte para aniquilarlas (Mojica, 1999, p. 6).

En la percepción esperada o prospectiva 2019 - 2030 del Factor Total Gestión, Implementación y Desempeño del SNIA, la percepción positiva o real está por arriba del valor teórico, lo que significa que se percibe de manera general un SNIA más allá de lo aceptable, no obstante, no supera el percentil 70 propuesto como el mínimo esperado para un SNIA con expectativas más desafiantes. En este sentido se encontró que el Factor 1 ASTIA, el Factor $5 \mathrm{y}$ el Factor 8 son los que mejor valor real presentan de $69.06 \%, 43.60 \%$ y $30 \%$ respectivamente, se encuentran en la franja superior al valor teórico y por debajo del nivel máximo esperado, pero sin superar el percentil 70 como mínimo esperado en el presente estudio.

La percepción esperada de todos los factores del SNIA se encontró diferencias altamente significativas en la visión de los actores respecto a la prospectiva total del SNIA (t:6.802; p-valor $=0.000)$. 


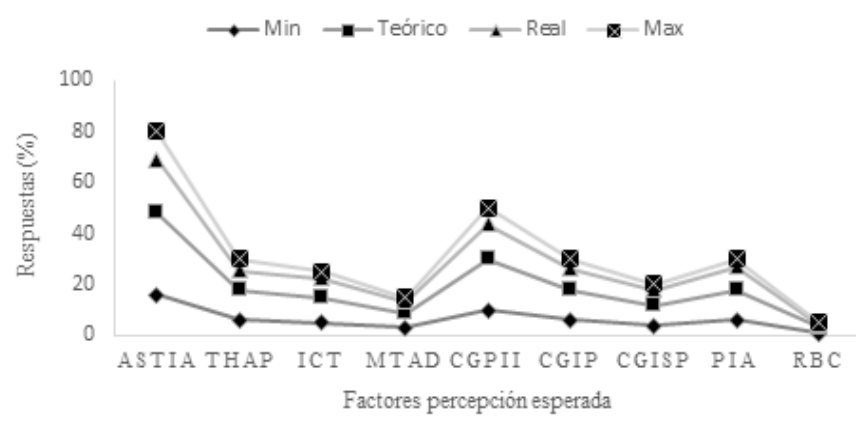

Figura 2. Percepción esperada o perspectivas del Sistema Nicaragüense de Investigación e Innovación Agropecuaria SNIA 2019 $-2021$.

ASTIA = Articulación del Sistema Territorial de Innovación Agropecuario, THAP $=$ Talento Humano en las Áreas Agropecuarias Prioritarias, $\mathrm{ICT}=$ Infraestructura Científica Tecnológica, $\mathrm{MTAD}=$ Mercados de Tecnologías Apropiadas y Disponibles, CGPII= Calidad en la gestión de los procesos de innovación de las instituciones de formación e investigación agropecuaria y áreas afines, CGIP= Calidad en la gestión de la innovación desde el sector público, $\mathrm{CGISP}=\mathrm{Calidad}$ en la gestión de la innovación en el sector de productores, $\mathrm{PIA}=$ procesos de innovación agropecuaria, $\mathrm{RBC}=$ Relación beneficio costo de los procesos de innovación agropecuaria.

\section{Análisis de la prospectiva del SNIA a partir de la per-} cepción esperada 2019 - 2021 vs la percepción actual 2019. Si queremos comprender cómo la globalización impactará el futuro de la extensión agropecuaria y forestal, no debemos mirar primero dentro de la agricultura o de la ganadería o de la silvicultura, o de la extensión sino ser prospectivos, e indagar primero sobre la naturaleza de fenómenos más amplios que afectarán al proceso de extensión. Analizar las capacidades y competencias del extensionista ante la globalización implica cuestionar también nuestra percepción sobre el medio rural y las comunidades que lo constituyen, sobre los actores y factores que intervienen en el desarrollo comunitario, implica también capacitarnos en métodos y técnicas de comunicación eficaces, conocer sobre la región y sus relaciones socio-económicas con el resto del país, y caracterizar el fenómeno de la globalización en el contexto de un cambio de época. (Russo, 2009, p. 90).

En Prospectiva, es decir la visión de futuro de los actores del SNIA reflejan como altamente relevante continuar trabajando en función de la articulación del Sistema Territorial de Innovación Agropecuario, la calidad en la gestión de los procesos de innovación de las instituciones de formación e investigación agropecuaria y áreas afines y finalmente en los procesos de innovación agropecuaria.

Es por ello que el rol de coordinación del SNIA bajo el liderazgo del INTA - CNU - CONACOOP deben fortalecer la Secretaria Técnica del SNIA como un espacio que le permita al SNIA disponer de un espacio físico, visibilidad y sobre todo el espacio para planificar, implementar y gestionar los principales lineamientos de los procesos de innovación.

El análisis global del SNIA en la brecha positiva de la prospectiva 2019 - 2030 con relación a la retrospectiva 2015 - 2019, se encontró que de manera global los actores del SNIA hay tres factores que los valoran más alto tales como la Articulación del Sistema Territorial de Innovación Agropecuario Factor 1 ASTIA con el 14.62\%, la Calidad en la Gestión de los procesos de innovación de las instituciones de formación e investigación agropecuaria y áreas afines Factor 5 CGPII con el 11.68\% y los Procesos de Innovación Agropecuaria Factor 8 PIA con el 6.56\%. El indicador donde existe la menor percepción positiva como brecha es el el Factor 9 Relación Beneficio Costo RBC con un $0.98 \%$.

En este sentido los procesos de innovación agropecuaria y su gestión en el sector público y productivo requieren una especial atención del equipo de coordinación del SNIA desde una agenda novedosa que vincule a los diferentes actores del sector público, sector científico - tecnológico y el sector productivo y sabiduría popular; esta vinculación podrá ser posible aprovechando la fortaleza organizativa de los sistemas de innovación territorial a nivel de los Núcleos de Investigación e Innovación Territorial NITs y Grupos de Innovación Agropecuaria GIAs, los cuales son reflejados en esta investigación como la clave del éxito en los territorios.

Al realizar MANOVA de los nueve factores totales del estado actual del SNIA como variables dependientes y utilizando como factor fijo el nombre o razón social en la que labora el encuestado cuya etiqueta es Sector Productivo, Sector Público y Sector Científico - Tecnológico, se encontró que la prueba de Lambda de Wilks con un p-valor $=0.334$ destacando que los sectores productivos, científico - tecnológico y sector público no ven diferencias en los nueve factores considerando que la valoración está hecha en una franja que no supera el percentil 70.

Crear ambientes innovadores es una tarea impostergable en los territorios. Para ello, se requiere construir procesos de aprendizaje que fortalezcan aspectos intangibles como el valor de las relaciones reciprocas generalizadas (forma de capital social) y la gestión del conocimiento en sus dimensiones tácito, codificado y sus relaciones virtuosas. La Red de Gestión del Conocimiento para el Desarrollo Rural de Matagalpa y Jinotega es un espacio de múltiples actores que apuesta al fortalecimiento del capital 
social territorial y a la promoción de ambientes apropiados para el aprendizaje colectivo de las vías más apropiadas para el desarrollo, ubicando el conocimiento en el centro de su accionar. Tenemos el reto colectivo de avanzar, reflexionar acerca de nuestro accionar y re direccionar si es necesario, para luego reflexionar sobre nuestro nuevo accionar, en una especie proceso dialéctico (Rojas \& Espinoza, 2013, p. 48).

\section{Impactos potenciales del SNIA en las dimensiones so-} cial, económica, ambiental y organizacional. Las economías de América Latina y el Caribe (ALC) presentan un severo déficit en cuanto a la incorporación de conocimiento y tecnología a sus procesos productivos. Es común la creencia de que este déficit de innovación se puede atribuir en parte a la concentración de la estructura productiva de la mayor parte de los países de la región en la explotación de recursos naturales, un tipo de industria que tradicionalmente se ha considerado como de menor intensidad tecnológica. Si esta fuese toda la explicación, la noción de déficit de innovación sería discutible. Simplemente, el bajo nivel de intensidad tecnológica sería el producto espontáneo del funcionamiento de economías que han desarrollado un cierto tipo de especialización en el contexto de la economía mundial. Sería un equilibrio que puede o no agradar a la percepción generalizada de la importancia de la tecnología como fuente principal del dinamismo económico, pero un equilibrio al fin y al cabo (Navarro \& Olivari, 2016, p. 4).

Los impactos del SNIA se orientan a diferentes dimensiones y se destacan tres dimensiones con las percepciones más bajas desde los actores del SNIA entre las que se destacan el Fortalecimiento del enfoque mujer y juventud e interculturalidad con el $88.89 \%$, estrategia de aprovechamiento ambiental con un $93.65 \%$ y la eficiencia tecnológica con un $93.65 \%$; no obstante un segundo bloque que presenta percepciones bajas están el referido a la generación de beneficios económicos con un $96.83 \%$ y la política de innovación agropecuaria con un $96.83 \%$.

Los recursos sociales, son aquellos recursos que poseemos a partir de las relaciones [familiares, comunitarias, de amistad, de trabajo o de negocio] con otras personas o grupos; así como por nuestra afiliación a diferentes tipos de organizaciones [gremiales, de productores, políticas, comunitarias, religiosas, entre otras]. Este tipo de recursos permite desarrollar estrategias para el logro de los medios de vida [que requieren de coordinación y acción colectiva] y facilita el acceso a otros recursos esenciales para el logro de los medios de vida (Gottret, 2011, p. 20).
El impulso por parte del SNIA de una política de innovación agropecuaria en el país es una dimensión económica que se relaciona significativamente con el SNIA como una oportunidad para fortalecer un modelo de innovación basado en redes con enfoque sistémico como una dimensión organizacional. $(\mathrm{p}=0.000<0.05)$.

Como el coeficiente de contingencia es menor que $0.05(0.000<0.05)$ rechazamos la hipótesis nula y aceptamos la hipótesis alternativa, luego podemos concluir que a un nivel de significancia de 0.05 , existe una relación fuerte entre el impulso por parte del SNIA de una política de innovación agropecuaria en el país es una dimensión económica y el SNIA como una oportunidad para fortalecer un modelo de innovación basado en redes con enfoque sistémico como una dimensión organizacional.

La eficiencia de los sistemas de innovación depende de la posibilidad de elevar y/o consolidar las capacidades de cada uno de los actores. Las facultades del sistema se desarrollan en mayor o menor medida por el grado de alineación o acuerdo entre los actores para establecer la visión del mismo: hacia dónde va el sistema; cuáles son sus objetivos; qué es lo que se quiere lograr específicamente; qué mecanismos se requieren para alcanzarlos, y cómo se van a alcanzar los objetivos si se es coherente con la visión establecida. El análisis del SNIN permite agruparlo en los siguientes cuatro subsistemas: el sector productivo, el sector gobierno, las universidades y centros de investigación, y los organismos no gubernamentales (CONICYT, 2010, p. 30).

La causalidad de esta relación entre el desempeño económico y las actividades de Ciencia Tecnología e Innovación CTI es compleja, presenta una doble direccionalidad con rezagos temporales significativos y no siempre es posible establecer en torno a ella vínculos lineales directos. Sin embargo, dada la importancia económica adquirida por la CTI, los crecientes esfuerzos realizados para su desarrollo, particularmente en los países más avanzados y las economías emergentes, y las oportunidades que representan en el actual contexto internacional, es necesario reflexionar sobre las distintas dimensiones de esta relación, tanto por sus implicancias efectivas como potenciales (Dutrénit et al. 2010, p. 21).

Las principales brechas percibidas por los actores del SNIA se alejan de un impacto más inmediato especialmente con indicadores como la eficiencia tecnológica, estrategia de aprovechamiento ambiental, generación de beneficios económicos, política de innovación agropecuaria, modelos basados en redes de innovación; lo cual im- 
plica al SNIA un mayor nivel de gestión, implementación y desempeño de cara a los próximos años.

Los avances científicos y tecnológicos del mundo en las últimas décadas han hecho que las ventajas comparativas de los países basadas en su ubicación geográfica o en su dotación privilegiada de recursos naturales hayan dado paso a ventajas competitivas sustentadas en la generación y aplicación del conocimiento.

La sociedad del conocimiento, así denominada, ha determinado profundos cambios institucionales, socioeconómicos y científicos, en los cuales la educación y la conformación de verdaderos sistemas de ciencia, tecnología e innovación son indispensables para estructurar y soportar los planes nacionales de desarrollo. Estados avanzados de desarrollo requieren sistemas bien estructurados que integren todos los actores relacionados con la academia, la ciencia, la tecnología y la innovación, con una visión compartida y un enfoque proactivo. No hacerlo significaría un retroceso representado en la pérdida de mercados internacionales, la entrega de mercados nacionales, el aumento de la dependencia política y económica y el rápido e inevitable empobrecimiento de la población (Monroy, 2006, p. 158).

\section{Prioridades del SNIA a nivel nacional, regional y terri-} torial. Es necesario aclarar que la agricultura en Nicaragua es fundamentalmente para garantizar la seguridad alimentaria y la sobrevivencia de la nación, en este sentido; suelo, agua, bosques y aire son bienes públicos, pero hasta ahora su uso ha tenido carácter privado y dichos bienes, no son considerados como asunto de seguridad nacional. Ante el agotamiento de los recursos y la insostenibilidad de nuestra actual forma de producir, todas las organizaciones públicas y privadas y los ciudadanos nicaragüenses debemos asumir el compromiso de promover otras formas de agricultura que produzcan y a la vez restauren y conserven los bienes naturales (Landero, Obando, Salmeron, Valverde y Vivas, 2016, pp. 17-18).

Las prioridades del SNIA son en realidad los retos y desafíos del país, en términos de incremento de la productividad en condiciones de variabilidad climática, con enfoque agroecológico, cuido de la madre tierra y el medio ambiente; no obstante, no es posible priorizar de manera genérica, y es por esto que los actores del SNIA al analizar las prioridades a nivel nacional, regional y territorial.

El desarrollo rural territorial desde los actores sociales, particularmente de la agricultura familiar, busca desplegar los recursos potenciales de carácter endógeno, en la definición de objetivos propios de desarrollo y la construcción y apropiación del territorio. Esta perspectiva se puede vincular y coordinar con políticas públicas de desarrollo rural para propiciar el fortalecimiento de los activos con los que cuentan la agricultura familiar y el desarrollo rural (Ramírez-Juárez, 2013, p. 36).

Los resultados sobre las prioridades del SNIA al nivel nacional, destacan dos lineamientos estratégicos para el corto plazo 2019 - 2021; con el $63 \%$ se prioriza la estrategia de productividad y competitividad del país según potencialidades y con el $65 \%$ para continuar con la Política de Investigación e Innovación Tecnológica.

En cuanto a los lineamientos para el largo plazo 2021 - 2030, se encontró que los actores del SNIA han priorizado para el nivel nacional con el $56 \%$ la gestión de indicadores de innovación y producción científica del país y con el $62 \%$ para las competencias de capacidades estratégicas de trabajo en redes.

A nivel de los Consejos Regionales de Investigación e Innovación Agropecuaria CRIAs, los actores del SNIA consideran que el fortalecimiento de capacidades de gestión de los Núcleos de Innovación Territorial NITs es de alta prioridad para el SNIA, seguido de la gestión de indicadores de innovación de procesos, productos y servicios para el nivel territorial, el desarrollo de un mecanismo funcional de seguimiento y evaluación a los planes territoriales.

Las prioridades para los actores del SNIA, se encontró para el corto plazo 2019 - 2021 dos principales prioridades, entre ellas, con el $62 \%$ se prioriza el desarrollo de un mecanismo funcional de seguimiento y evaluación a los planes territoriales y con el $75 \%$ para el fortalecimiento de capacidades de gestión de los Núcleos de Innovación Territorial NITs.

Las prioridades del SNIA a nivel regional tienen coherencia con lo planteado por (Rojas Meza, 2015, p. 45) el cual da a conocer que los sistemas territoriales de innovación agropecuaria deben responder a las particularidades de cada territorio y específicamente a una agenda de innovación, a las formas de organización de los productores, del sector público, los gobiernos municipales, las instituciones de formación e investigación y todas las expresiones de capital social que pueden incidir en el proceso de transformación de la agricultura.

La función primordial de la agricultura sigue siendo la producción de alimentos y de otros productos básicos, y contribuir a la seguridad alimentaria, tarea compleja que exige un medio ambiente favorable y políticas que garanticen la estabilidad y equidad social, cultural, 
política y económica. La combinación de las funciones económica, social y ambiental de la agricultura puede contribuir al cumplimiento de esos objetivos. La actividad agrícola y la utilización correspondiente de las tierras también generan una amplia gama de productos y servicios no alimentarios, configuran el medio ambiente, afectan a los sistemas sociales y culturales y contribuyen al crecimiento económico (FAO, 1999, p. iii).

El SNIA a nivel territorial según los actores del mismo, se encontraron tres lineamientos para el corto plazo de alta prioridad, con el $79 \%$ se priorizó la agenda de investigación e innovación territorial articulada, con el 62 $\%$ para la difusión de innovaciones, resultados y conocimientos y con el 67\% para la continuidad con los Grupos de Innovación Agropecuaria por Rubros según potencialidades territoriales.

En cuanto a las prioridades del SNIA para el nivel territorial de largo plazo 2021 - 2030 se encontró que hay dos prioridades bien definidas, con el 57\% para los mecanismos de aprendizajes y con el 56\% para las competencias de capacidades estratégicas de trabajo en redes.

Según Barreto "la agricultura familiar es una actividad clave en la reactivación de las economías rurales, generando estabilidad y arraigo social y nuevos horizontes de desarrollo, sobre todo para la juventud rural“ (2015).

Sumándole al planteamiento de la apropiación del territorio, la cooperación es fundamental para poder dar los saltos cualitativos que serán necesarios desde procesos de innovación social para enfrentar innovaciones tecnológicas más eficaces y eficientes.

Construyendo lazos de confianza, fomentando procesos de cooperación interempresarial y de los sectores empresariales con otras organizaciones públicas, privadas y la comunidad; impulsando procesos evolutivos de especialización e innovación a partir de compartir conocimientos y el reforzamiento de competencias; identificando escenarios e impactando socialmente, se puede mejorar el desempeño de las empresas y del territorio en su conjunto (Narváez y Fernández, 2008, pp. 74-75).

La alta prioridad de mediano a largo plazo planteada por los actores del SNIA referente a la continuidad con los Grupos de Innovación Agropecuaria por rubros según potencialidades territoriales.

El desarrollo de los territorios y sujetos rurales necesita de nuevas formas de abordar la compresión de sus problemáticas y posibilidades de desarrollo. En un territorio de Nicaragua la universidad y una organización campesina llevan desde el 2013 construyendo un proceso de diálogo transdisciplinario para generar estrategias de desarrollo des- de las familias de trece comunidades rurales. ¿Permitirá este diálogo identificar elementos del Buen Vivir en el futuro del campesinado en este territorio rural? (Alfaro-Mardones, Fernández-Hernández, y González-García, 2015, p. 16).

Los procesos participativos son esenciales, ya que ninguno de los múltiples actores y administraciones dispone de la autoridad, el conocimiento o los recursos para imponer o llevar adelante unilateralmente la estrategia para el territorio por él deseada. La estrategia territorial más efectiva será aquella que combine los aspectos positivos de la aproximación clásica (el análisis consciente y racional, apoyado por ejercicios de prospectiva y escenarios futuros) con el de la aproximación procesual (con espacios para la discusión y negociación que permitan aprendizajes y la generación de visiones compartidas) (Navarro-Aranguren, y Margo, 2012, p. 43).

Los cambios requeridos no se logran solo a través de decisiones unilaterales de política gubernamental, por vigorosas que estas sean. Por lo general, las reformas exitosas son resultado de acuerdos que han logrado movilizar a los principales actores para que participen del esfuerzo requerido, asegurándoles que los beneficios resultantes serían también compartidos. Para que Chile dé un salto a través de la innovación necesita movilizar y reclutar las voluntades de los actores relevantes -gobierno, empresas, trabajadores, científicos y educadores, entre otros-. Alcanzar un acuerdo amplio posibilitará que las reformas que se implementen generen un impacto significativo y equitativo en el desarrollo del país (Tokman y Zahler, 2004) .

Por consiguiente, se hace necesario al nivel territorial trabajar modelos integradores específicos buscando la especialización en el desarrollo de acciones de investigación, innovación y transferencia de tecnologías, con planes específicos y coherentes con el pensamiento sistémico estratégico y operativo para actuar como plataformas especificas territoriales en la búsqueda de lograr una armonía entre las necesidades (demanda) y la oferta de servicios, productos, prácticas y tecnologías en el mercado de servicios territoriales.

\section{CONCLUSIONES}

Actualmente el SNIA no ha logrado desarrollar su función principal como sistema de innovación desde los niveles territorial, regional y nacional mediante un proceso de la planificación, implementación, seguimiento, evaluación y sistematización de las acciones de investigación e innovación agropecuaria.

El Sistema Nicaragüense de Investigación e Innovación Agropecuaria SNIA no supera el percentil 70 como 
el mínimo esperado para ser un SNIA aceptable; lo que indica que las brechas del SNIA para lograr una gestión, implementación y desempeño son una tarea urgente para las instituciones o instancias nacionales de coordinación como el INTA, CNU y CONACOOP.

Los factores que marcan diferencias en el SNIA están referidos a la Articulación del Sistema Territorial de Innovación Agropecuario, la calidad en la gestión de los procesos de innovación de las instituciones de formación e investigación agropecuaria y áreas afines y los procesos de innovación agropecuaria; siendo el factor que más se aproxima al límite inferior, pero de mucha importancia es la relación beneficio costo de los procesos de innovación agropecuaria.

Los principales impactos percibidos por los actores del SNIA se relacionan con la participación de actores en la gestión de la innovación, el SNIA como referencia en indicadores de investigación e innovación, liderazgo del sector productivo, y generación de tecnologías sostenibles; y donde menor percepción de impacto del SNIA es en el fortalecimiento del enfoque mujer, juventud e interculturalidad.

Dentro de las prioridades identificadas se destacan al nivel nacional la política de gestión y acceso a recursos para facilidades de innovación, investigación (fondos competitivos para iniciativas territoriales), al nivel regional el fortalecimiento de capacidades de gestión de los Núcleos de Investigación e Innovación Territorial NITs y a nivel territorial se logran dos grandes prioridades como son la agenda de investigación e innovación territorial articulada y continuar con los Grupos de Innovación Agropecuaria por Rubros según potencialidades territoriales.

Es recomendable para el equipo de coordinación del SNIA (INTA, CNU y CONACOOP) impulsar una estrategia de fortaleciendo a los Núcleos de Investigación e Innovación Territorial NITs y Grupos de Innovación Agropecuaria GIAs como espacios generadores de la agenda nacional del SNIA donde se incluyan las prioridades a través de un plan con enfoque prospectivo para los próximos años con metas e indicadores bien definidos y un sistema de medición y aprendizajes que permita un proceso de institucionalización del SNIA.

El SNIA debe contar con una Secretaría Técnica que se instale como un espacio físico para impulsar los procesos de articulación de los diferentes actores del país, desde los diferentes niveles organizativos del SNIA basados en el triángulo científico tecnológico, integrando actores del Sector Publico, Sector Productivo, Sector Científico Tecnológico en todos los espacios de coordinación con énfasis en los territorios específicos según sus potencialidades, necesidades, características socio productivas y agroecológicas.

\section{REFERENCIAS BIBLIOGRÁFICAS}

Alfaro Mardones, J. I., Fernández Hernández, C. d., y González García, M. d. (2015). La transdisciplinariedad una herramienta para apuntar al Buen Vivir. Polis, Revista Latinoamericana, 23-42.

Barreto, G. (2015). Desarrollo Rural para la erradicación del hambre en Nicaragua. Desarrollo Rural para la erradicación del hambre en Nicaragua. León.

Boisier, S. (2005). ¿Hay espacio para el desarrollo local en la globalización? Revista de la CEPAL 86, 16.

Briones, M. A., Monterrey, J., Orozco, F., Vera, T., Romero, B., y Jennifer, A. (2015). Catálogo de tecnologías agropecuarias en Nicaragua. Managua: FUNICA.

Canales, F., Alvarado, E., y Pineda, E. (1994). Metodología de la investigación: Manual para el desarrollo de personal de salud. Washington: Organización Panamericana de la Salud.

Casado Cañeque, F. (2007). Alianzas público - privadas para el desarrollo. Madrid: Fundación Carolina - CeALCI.

CONICYT. (2010). Plan Nacional de Ciencia, Tecnología e Innovación Nicaragua, 2010 - 2013. Managua: CONICYT.

Dutrénit, G., Capdevielle, M., Corona, J., Puchet, M., Santiago, F., y Vera, A. (2010). El Sistema Nacional de Innovación Mexicano: Instituciones, Políticas, Desempeño y Desafios (Primera ed.). Coyoacán, Mexico: Universidad Autónoma Metropolitana. Obtenido de https://mpra.ub.uni-muenchen.d/31982/

FAO. (12-17 de Septiembre de 1999). Documento preparado para la conferencia FAO / Paises Bajos sobre El Caracter multifucional de la agricultura y la tierra. Maastricht, Paises Bajos.

García, D., Gálvez, E., y Maldonado, G. (2016). Efecto de la innovación en el crecimiento y el desempeño de las MIPYMES de la Alianza del Pacífico. Un estudio empírico. Universidad ICESI: Estudios Gerenciales(32), 326-335.

Gottret. (2011). El enfoque de medios de vida sostenibles: Una estrategia para el diseño e implementación de iniciativas para la reducción de la pobreza. Turrialba-Costa Rica: CATIE.

Hernández, R., Fernández, C., y Baptista, M. d. (2014). Metodología de la investigación . Mexico: Mc Graw Hill Education.

Landero, B., Obando, S., Salmeron, F., Valverde, L., y Vivas, E. (2016). Agricultura sostenible para enfrentar los efectos del cambio climático en Nicaragua (1A ed. ed.). (V. V. Elgin Antonio, Ed.) Managua, Nicaragua: Fundación Friedrich Ebert. 
Mojica, F. J. (1999). Determinismo y Construcción del futuro. Consejo Americano para la Universidad de las Naciones Unidas, Bogota. Recuperado el 25 de Abril de 2019, de http://www.celgyp.org/trabajos/trabajos/Determinismo_y_Construccion_del_Futuro.pdf

Monroy, S. E. (Julio a Diciembre de 2006). Nuevas políticas y estrategias de articulación del sistema de ciencia, tecnología e innovación colombiano. INNOVAR, 16(28), 157-172.

Narváez, M., y Fernández, G. Y. (2008). El desarrollo local sobre la base de la asociatividad empresarial: una propuesta estratégica. Opción, 74-92.

Navarro, J. C., Y Olivari, J. (2016). La política de innovación en América Latina y el Caribe: nuevos caminos. Washington, DC: BID.

Navarro, M., Aranguren, M.-J., y Margo, E. (2012). Las estrategias de especialización inteligente: una estrategia territorial para las regiones. Cuadernos de Gestión, 12, 27-49. doi:10.5295/cdg.110310mm

Piura López, J. (2012). Metodología de la investigación científica: Un enfoque integrador. Managua.

Ramírez Juárez, J. (2013). Transformaciones Agrarias y Desarrollo Rural Territorial. Matagalpa: Red de Gestión del Conocimiento para el Desarrollo Rural de Matagalpa y Jinotega.

Rojas Meza, J. Medición de la innovación agropecuaria desde los territorios: una propuesta conceptual y metodológica. La Calera, $15(24), 40-48$.

Rojas, J., y Espinoza, E. (2013). Desarrollo Rural Territorial: Enfoques, Metodologías y Experiencias. Managua.

Russo, R. O. (2009). Capacidades y Competencias del Extensionista Agropecuario y Forestal en la Globalización. Revista Comunicación, 86-91.

Tokman, M., Y Zahler, A. (2004). Innovación para un crecimiento sostenido: Siete lecciones para Chile. en foco: EXPANSIVA, 26. 